\title{
ASPECTOS MORFOLÓGICOS EN TOMOGRAFÍA COMPUTADA DE UN QUISTE DE DUPLICACIÓN INTESTINAL EN ADULTO: REPORTE DE CASO CLÍNICO Y REVISIÓN DE LA LITERATURA
}

Drs. Cristián Varela $U^{(1)}$, Ignacio Maldonado Sch ${ }^{(2)}$, Giancarlo Schiappacasse $F^{(3)}$, Alvaro Postigliatti $F^{(2)}$, Marcela Moreno $\mathbf{S}^{(4)}$.

1. Radiólogo Clínica Dávila, Docente Universidad de los Andes y Universidad Mayor. Santiago, Chile.

2. Becado de Radiología Universidad de Chile, Hospital San Juan de Dios. Santiago, Chile.

3. Radiólogo Clínica Alemana de Santiago, Docente U. de Chile Hospital San Juan de Dios. Santiago, Chile.

4. Anatomopatóloga, Docente U. de Chile Hospital San Juan de Dios. Santiago, Chile.

\section{COMPUTED TOMOGRAPHIC APPEARENCE OF ENTERIC DUPLICATION CYST IN ADULT PATIENT: A CASE REPORT AND LITERATURE REVIEW}

\begin{abstract}
Enteric duplication cysts are uncommon congenital anomalies of the gastrointestinal tract that may arise anywhere along the digestive tract. They most frequently occur in the small intestine (terminal ileum) on its mesenteric border and rarely communicate with the digestive tract. Enteric duplication cysts are usually asymptomatic and diagnosed during childhood (first 2 years of life). We report a case of unusual presentation in an asymptomatic 21-year-old patient, who was diagnosed by contrast-enhanced multislice computed tomography. Keywords: Computed tomography, Enteric duplication cyst.
\end{abstract}

Resumen: Los quistes de duplicación intestinal, representan anomalías congénitas del tracto gastrointestinal poco frecuentes. Estos pueden surgir en cualquier localización a lo largo del tubo digestivo. La mayoría se desarrollan en el intestino delgado (lleon terminal) en su borde mesentérico y rara vez presentan comunicación con el tracto digestivo. Usualmente, son diagnosticados durante la infancia (primeros 2 años de vida) donde suelen ser sintomáticos. Se expone un caso de presentación poco usual, en un paciente de 21 años, asintomático previamente, donde se llegó al diagnóstico a través de la tomografía computada multicorte contrastada.

Palabras Clave: Tomografía computada, Quiste de duplicación intestinal.

Varela C. Aspectos morfológicos en tomografía computada de un quiste de duplicación intestinal en adulto: Reporte de caso clínico y revisión de la literatura. Rev Chil Radiol 2011; 17(1): 30-34.

Correspondencia: Dr. Cristián Varela U. / cvarelau@vtr.net

Trabajo recibido el 08 de marzo de 2010, aceptado para publicación el 19 de enero de 2011.

\section{Introducción}

Los quistes de duplicación intestinal representan anomalías congénitas poco frecuentes del tubo digestivo. Pueden surgir en cualquier localización a lo largo del tracto gastrointestinal, la mayoría se sitúan en esófago distal e lleon terminal, clásicamente en el borde mesentérico. Generalmente son estructuras esféricas o tubulares, que rara vez se comunican con el tracto gastrointestinal.

Se reporta un caso de quiste de duplicación entérico del ileon distal en un adulto, diagnosticado con tomografía multicorte contrastada (TCMC).

\section{Caso Clínico}

Paciente sexo femenino, con antecedentes de dolicomegacolon y quiste mesentérico operado a los 27 días de vida. Asintomática hasta los 21 años de edad, cuando presenta dolor abdominal tipo cólico periumbilical recurrente, vómitos postprandiales, baja de peso, 4 Kilos en 2 meses, asociada a leve anemia ferropénica refractaria a tratamiento (Hto $31 \%$ ). Los estudios de ultrasonografía abdominal y ginecológica, además de la endocospía digestiva alta fueron normales; la enema baritada sólo reportó dolicomegacolon. 
En el laboratorio fueron negativos: test de hemorragia oculta en deposiciones, coprocultivo, parasitológico seriado y estudio para enfermedad celíaca.

En la última consulta, el médico tratante palpó una masa abdominal, motivo por el cual solicitó TCMC de abdomen y pelvis. Este estudio demostró una gran lesión intraperitoneal de aspecto entérico dependiente del meso del intestino delgado, sugerente de quiste de duplicación intestinal (Figuras 1,2), planteándose como diagnóstico alternativo un divertículo de Meckel gigante.
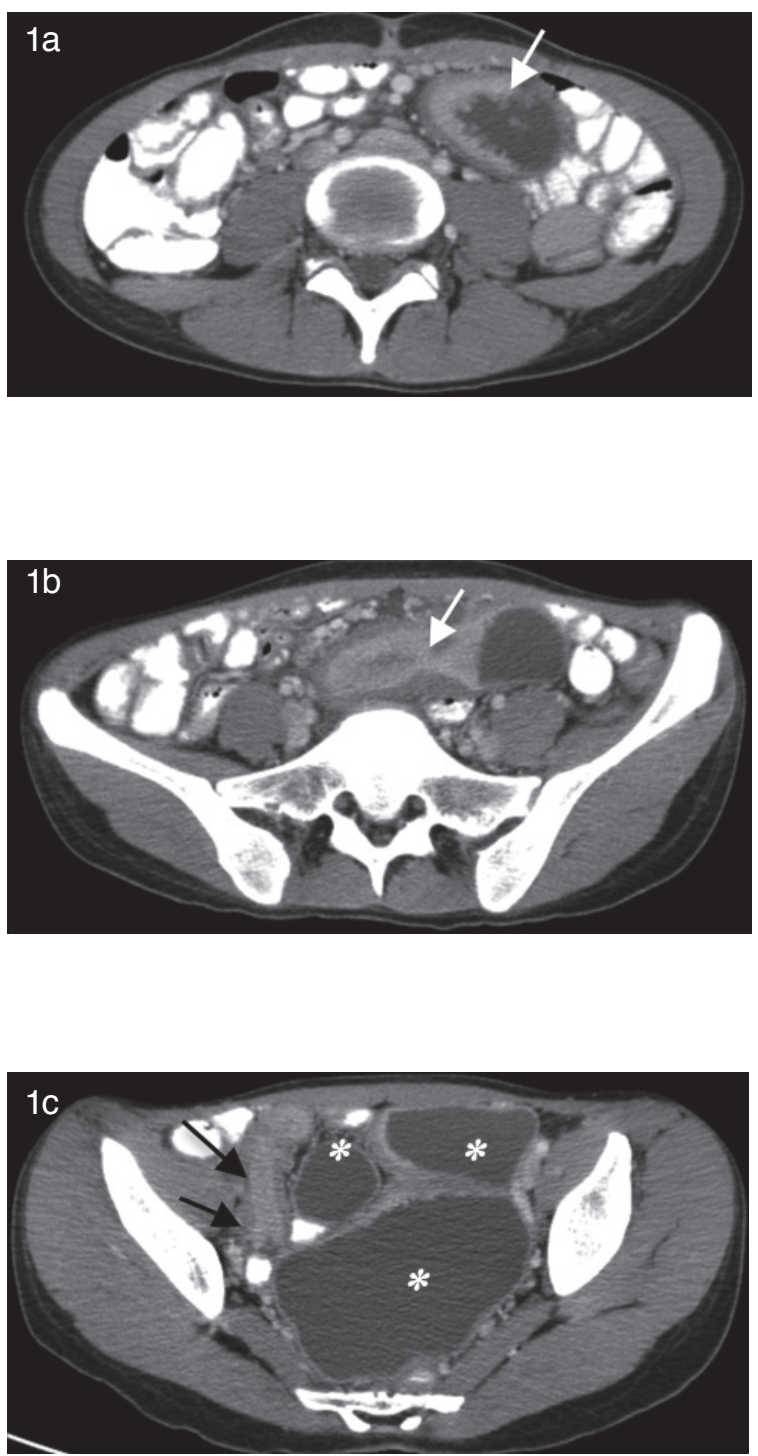

Figura 1. Cortes axiales con contraste oral y ev, en fase portal. a) Masa intraperitoneal en flanco izquierdo con gruesa pared estratificada, mucosa de aspecto polipoídeo y contenido líquido sin contraste en su interior; b) Corte axial más caudal en pelvis mayor: continuidad de la estructura hacia cavidad pélvica, de morfología tubular (flecha); c) Corte axial en pelvis menor: lesión con morfología saculary abundante contenido líquido $\left(^{*}\right)$. Flechas muestran aparente comunicación con ileon terminal.
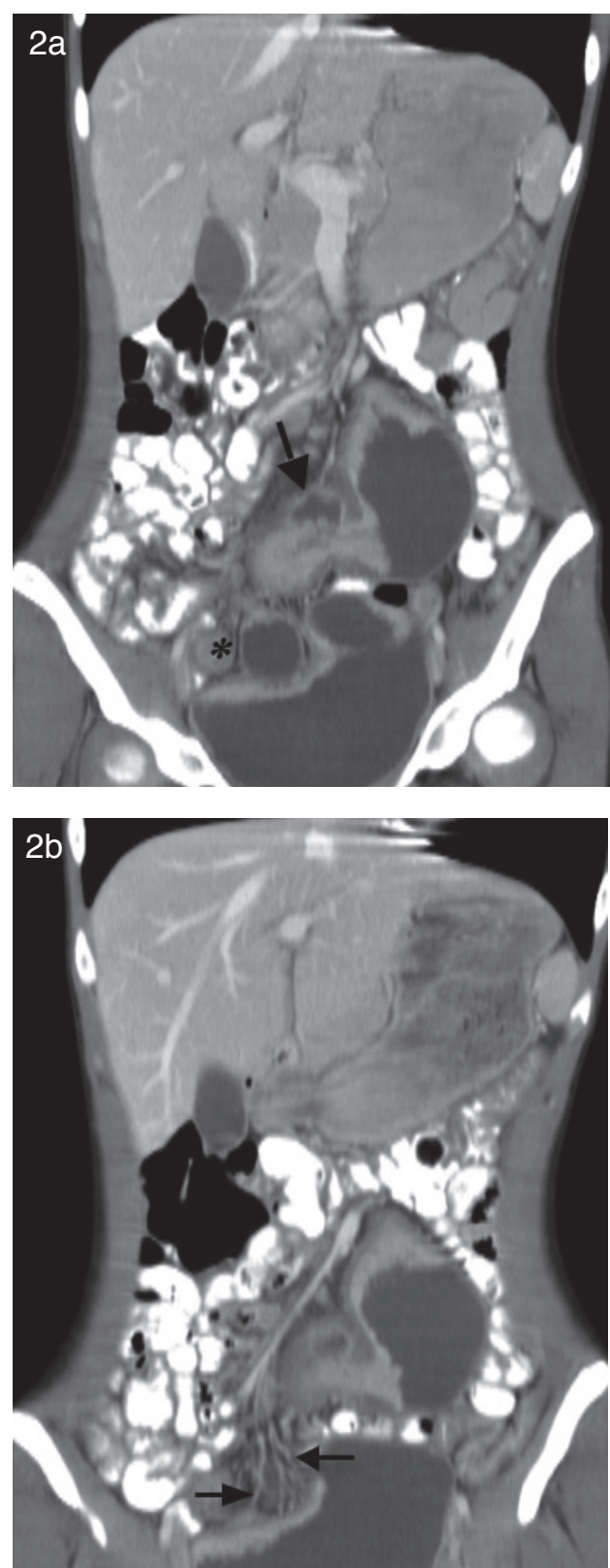

Figura 2. Reconstrucciones coronales (MPR) con contraste oral y ev. a) Visión panorámica de la voluminosa lesión intraperitoneal, que ocupa casi completamente la pelvis, con pared de aspecto enteral. Imagen con saliente de perfil, posible úlcera. La estructura presenta íntima relación con el mesenterio, unida a distal con la pared del ileon terminal. b) Se demuestra estructura irrigada por vasos dependientes de la raíz del mesenterio.

Estando en espera de cirugía electiva, sufre cuadro de obstrucción intestinal, por lo que es sometida a laparotomía exploradora, que demostró obstrucción intestinal secundaria a masa de $40 \mathrm{~cm}$ de longitud en ileon terminal, a $50 \mathrm{~cm}$ de válvula ileocecal, que ocupaba la excavación pélvica comprimiendo las asas de intestino delgado y colon sigmoides; se efectuó resección de la masa según se observa en Figura 3. 


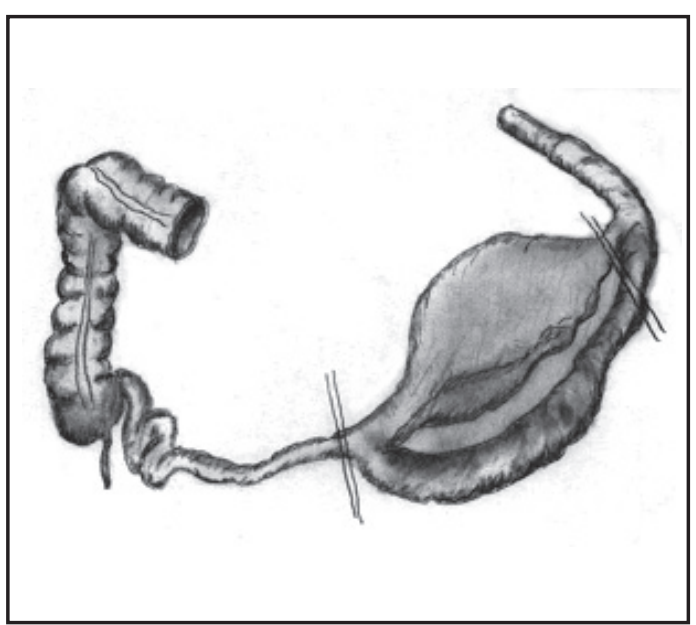

Figura 3. Esquema de protocolo quirúrgico.

La anatomía patológica confirmó que se trataba de un quiste de duplicación intestinal, con presencia de mucosa gástrica ectópica y úlcera crónica activa en el fondo del asa intestinal duplicada (Figuras 4,5)(4).
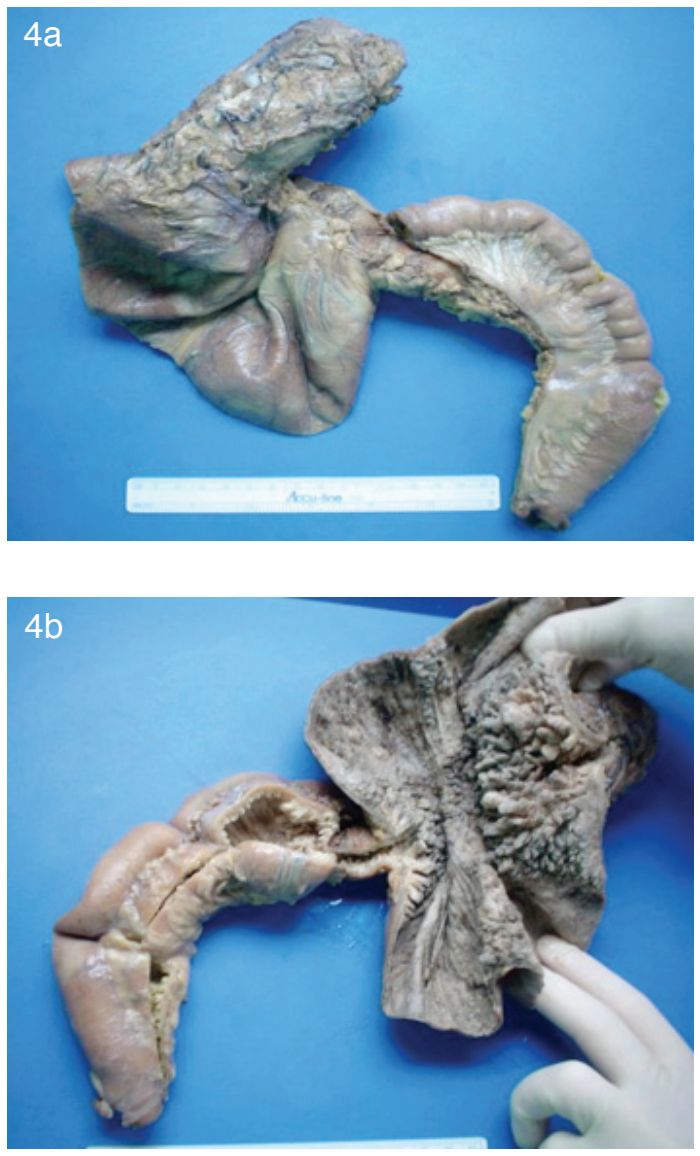

Figura 4. a) Pieza quirúrgica demuestra masa adyacente al ileon terminal resecado; b) Quiste de duplicación abierto, con pliegues de mucosa gástrica ectópica.
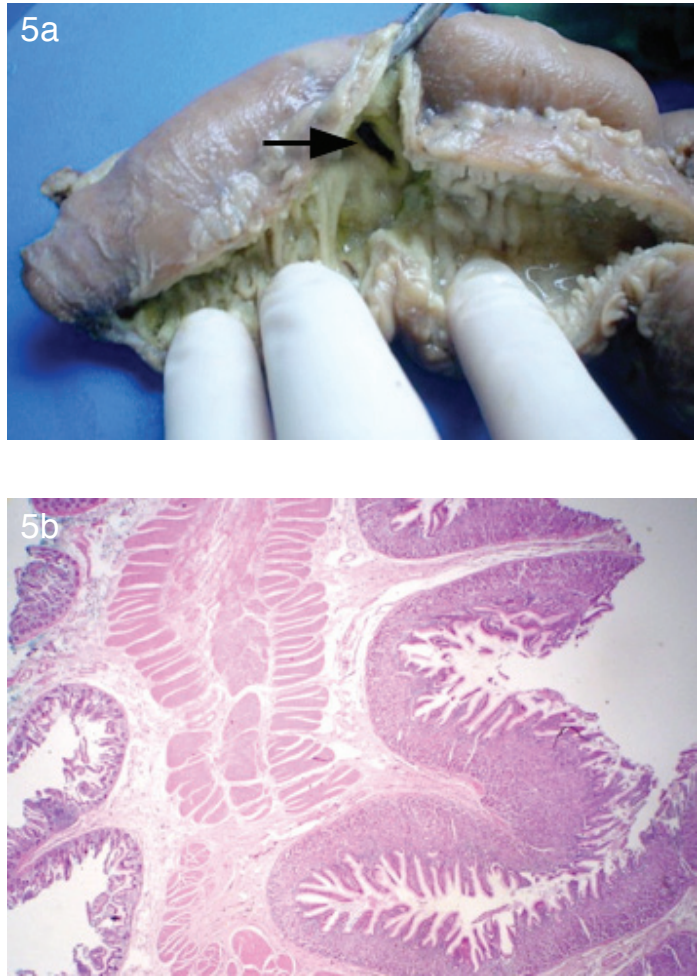

Figura 5. a) Ulcera crónica activa, perforada en lumen de asa duplicada y comunicada a asa normal; b) Extensa metaplasia de mucosa de tipo gástrica en asa intestinal duplicada (derecha asa conservada, izquierda asa duplicada).

\section{Discusión}

Los quistes de duplicación entéricos corresponden a anomalías congénitas benignas de baja frecuencia. Desde el punto de vista anátomo-patológico se describen como estructuras esféricas o tubulares que se encuentran situadas en relación al borde mesentérico del intestino, compartiendo una pared muscular común y revestidos por mucosa digestiva, pudiendo presentar mucosa gástrica o tejido pancreático ectópico. Pueden desarrollarse en cualquier segmento a lo largo del tracto gastrointestinal, con mayor frecuencia en el ileon distal y esófago inferior. En general, el intestino delgado es la localización más frecuente, siendo en orden de frecuencia de mayor a menor: ileon, yeyuno y finalmente duodeno. Se han reportado otras localizaciones menos frecuentes y atípicas, tales como colon y páncreas, esta última simulando una imagen de pseudoquiste complicado con hemorragia secundaria a la presencia de mucosa gástrica ectópica ${ }^{(1)}$. Rara vez comunican con el tracto gastrointestinal.

La presencia de mucosa gástrica ectópica es variable, con incidencias reportadas en la literatura entre un $17-36 \%$, lo que explicaría el desarrollo de úlceras sobre la mucosa gástrica ectópica, hemorragia digestiva, invaginación y necrosis por compresión ${ }^{(2,3)}$ La presencia de mucosa pancreática ectópica, que suele ser más prevalente en los quistes de duplicación 
gástricos (37\%), pueden llegar a generar cuadros de pancreatitis con elevación de amilasas, perforación gástrica, con subsecuente extensión del proceso inflamatorio al páncreas adyacente, pudiendo enmascarar el origen de la pancreatitis aguda de tipo ectópica ${ }^{(1,2,4)}$.

La mayoría se presenta clínicamente durante el primer año de vida como masa palpable abdominal, distensión y dolor abdominal recurrente. Son escasos los casos reportados tardíamente que llegaron asintomáticos a la adultez ${ }^{(2,3,5-8)}$.

Las complicaciones descritas incluyen perforación, invaginación intestinal, vólvulo, obstrucción intestinal por compresión extrínseca de estructuras adyacentes, hemorragia digestiva y, en menor frecuencia, transformación maligna ${ }^{(2-4,6,9-11)}$.

Inoue y Nakamura reportaron 18 casos de transformación maligna de quistes de duplicación entéricos, con rango de edad entre 33 y 65 años, siendo el colon el segmento asociado a mayor malignización (67\%), tratándose principalmente de adenocarcinomas ${ }^{(3,11)}$.

Los quistes de duplicación también pueden asociarse aisladamente a otras malformaciones congénitas, principalmente vertebrales y genitourinarias ${ }^{(2,3,5,6)}$

Al estudio imaginológico, la ultrasonografía (US) es útil como aproximación inicial; la TCMC y la resonancia magnética $(\mathrm{RM})$ permiten esclarecer con mayor definición la anatomía y extensión de las lesiones, por las capacidades multiplanares de ambas técnicas y su evaluación panorámica de la cavidad peritoneal ${ }^{(2-4,6,10,12)}$.

La US es muy útil para demostrar la naturaleza quística de la lesión; el aspecto típico con este método consiste en una masa anecogénica, con refuerzo acústico posterior, en estrecha relación con el tracto digestivo, de morfología esférica o tubular, de paredes bien definidas. Se ha descrito el signo de la "doble pared" como característico a la US, permitiendo la diferenciación con otras lesiones de naturaleza quística; este aspecto de "doble pared" está dado por la imitación de la configuración normal del tracto digestivo a la US, con un anillo ecogénico interno, que corresponde a la interfase entre la mucosa-submucosa y un halo hipoecogénico externo correspondiente a la capa muscular (Figura 6). El contenido anecogénico puede aparecer heterogéneo, incluyendo material ecogénico móvil en su interior en caso de existir infección, hemorragia o comunicación con el tracto digestivo. El signo de la "doble pared" puede estar ausente en casos de infección, hemorragia y/o perforación ${ }^{(2,3,6,13)}$.

En TCMC, estas lesiones pueden presentar aspecto de masa quística adyacente al tracto gastrointestinal, que no realza con contraste intravenoso, en ocasiones con loculaciones en su interior. La mayoría de las veces, presentan atenuación de líquido, sin embargo ocasionalmente pueden alcanzar una mayor densidad

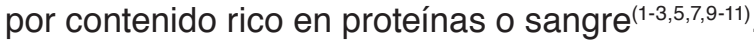

En RM por su naturaleza quística se comportan hipointensos en T1 e hiperintensos en T2, con realce

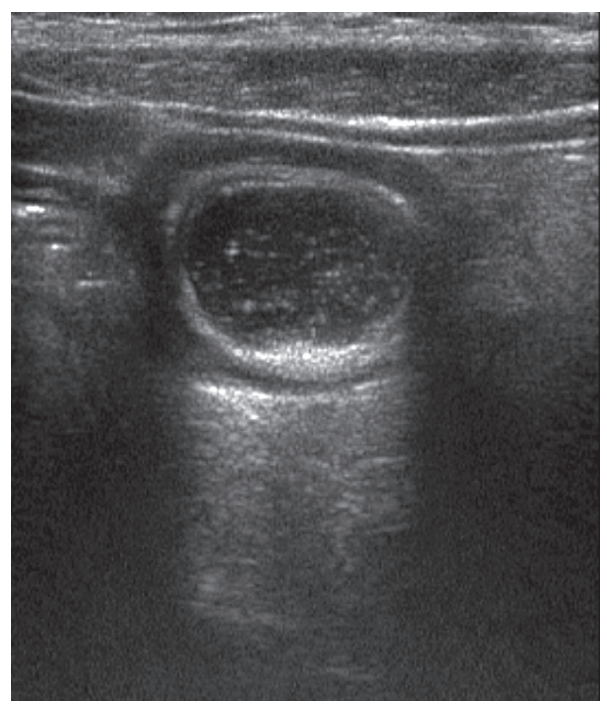

Figura 6. Imagen de quiste de duplicación intestinal en US, que corresponde a un paciente pediátrico.

anular; de existir hemorragia, infección o comunicación con el tracto gastrointestinal, la señal de T1 puede ser variable $(2,3,12)$.

En el caso de nuestra paciente, se trataba de una extensa lesión alargada tubular con algunas áreas más dilatadas, en íntima relación con el ileon. Su estructura estaba compuesta por una gruesa pared de varias capas, del todo similar al intestino. En el segmento de mayor diámetro, el revestimiento interno era de mayor grosor con un aspecto que recordaba al estómago, lo que coincidió con la mucosa gástrica demostrada en la patología. La clara dependencia de esta estructura del meso intestinal y de sus respectivas estructuras vasculares también fueron elementos que facilitaron el diagnóstico imaginológico.

En el diagnóstico diferencial se debe considerar: divertículo de Meckel, quistes mesentéricos o mesoteliales y linfangiomas quísticos. Dependiendo de su localización, también es posible considerar como alternativas: quiste de colédoco, pseudoquiste pancreático, quistes ováricos y mucocele apendicu$\operatorname{lar}^{(2,3,5,6,8,9,13)}$.

El tratamiento de elección es quirúrgico(5).

Desde el punto de vista clínico, el caso presentado es excepcional por su presentación tardía, considerando que la mayoría de los casos de duplicación intestinal se manifiestan y diagnostican en los primeros años de vida; existen escasos reportes de casos en adultos previamente asintomáticos. El intenso y recurrente dolor abdominal asociado a la presencia de anemia persistente se explicó por la existencia de mucosa gástrica ectópica en el quiste de duplicación, que se ulceró, sangró y posteriormente se comunicó con el lumen del asa intestinal normal, complicándose finalmente con el cuadro de obstrucción intestinal.

También es excepcional el presente caso desde la 
perspectiva del diagnóstico imaginológico, dado que se logró un diagnóstico no invasivo pre-quirúrgico con TCMC. La enema baritada no logró demostrar el quiste y sorprendentemente al US tampoco se pesquisó, siendo la TCMC la única técnica de imágenes que identificó y fue capaz de caracterizar correctamente la lesión intestinal.

\section{Bibliografía}

1. Grand DJ, Sobin LH, Fishman EK. Enteric duplication cyst of the pancreas: CT findings. Critical Reviews in Computed Tomography 2004; 45: 105-110.

2. Macpherson RI. Gastrointestinal tract duplications: clinical, pathologic etiologic and radiologic considerations; Radiographics 1993; 13: 1063-1080.

3. Tong SC, Pitman M, Anupindi SA. Best cases from the AFIP, lleocecal enteric duplicaction cyst: RadiologicPathologic Correlation; Radiographics 2002; 22: $1217-$ 1222.

4. Guarise A, Faccioli N, Ferrari M, Romano L, Parisi A, Falconi M. Duodenal duplication cyst causing severe pancreatitis: Imaging findings and pathological correlation. Worl J Gastroenterol 2006; 12(10): 1630-1633.

5. Donnelly LF. Fundamentals of pediatric radiology; Edit. Saunders 2001; Cap 5: 97-119.

6. Ranjeet SN, Jhanvi RR, Santosh JK, Arunima G, Priya
$\mathrm{H}$. Sonographic findings in a duodenal duplication cyst. J Clin Ultrasound 2002; 30(2): 566-568.

7. Thompson SK, Wong AL, Treneven CL, MacGregor JH. Enteric duplication cyst. Am J Surg 2004; 187: 316-318.

8. Menon P, Rao KLN, Chandirgarh KV. Isolated enteric duplication cysts; J Pediatric Surg 2004; 39(8): 5-7.

9. Hwang IK, Namkung S, Kim BS, Kim HC, Lee IS, Hwang WC. Perforated ileal duplication cyst with haemorrhagic pseudocyst formation. Pediatr Radiol 2003; 33: 489491.

10. Prada AM, Sanchís LS, Pérez CV, Wiehoff AN, Alonso LJ, Beltrá PR. Computed tomography diagnosis of symptomatic right paraduodenal hernia associated with enteric duplication cyst. J Pediatric Surg 2007; 42: 1938-1941.

11. Inoue $\mathrm{Y}$, Nakamura $\mathrm{H}$. Adenocarcinoma arising in colonic duplicaction cysts with calcification: CT findings of two cases. Abdom Imaging 1998; 23 : 135-137.

12. Wong AM, Wong HF, Cheung YCh, Wan YL, Ng KK, Koon-Kwan $\mathrm{Ng}$ et al. Duodenal duplication cyst: MRI features and the role of MR cholangiopancreatography in diagnosis. Pediatr Radiol 2002; 32: 124-125.

13. Cheng G, Soboleski D, Daneman A, Poenaru D, Hurbult $D$. Sonographic pitfalls in the diagnosis of enteric duplication cysts. AJR 2005; 184: 521-525.

\section{EN SALUD, \\ HAY MEJORES FORMAS DE AHORRAR.} Instala un dispensador Elite y ahorra en costo, no en calidad. 